\title{
Successful treatment of tabetic lightning pain and visceral crisis with gabapentin
}

\author{
Kyoko Oshita $\cdot$ Noboru Saeki $\cdot$ Hiroshi Niinai $\cdot$ \\ Hiroshi Hamada $\cdot$ Masashi Kawamoto
}

Received: 8 June 2011/Accepted: 16 August 2011/Published online: 29 September 2011

(C) The Author(s) 2011. This article is published with open access at Springerlink.com

Keywords Tabes dorsalis $\cdot$ Lightning pain · Gabapentin

To the Editor:

Lightning limb pain, a clinical feature of tabes dorsalis, is extremely severe and is refractory to various therapeutic approaches [1]. We report the case of a 61-year-old man who had severe paroxysmal lower limb pain for a decade because of tabes dorsalis. Nine years ago, he was diagnosed with tabes dorsalis and treated with high-dosage penicillin. The pain attacks were managed with carbamazepine. At the time of presentation, the patient complained of severe paroxysmal pain that was characterized by a pulling sensation extending from both legs up to the buttocks and which occurred three or four times daily. He writhed in pain, but apart from his pain attacks, he had no other complaints.

Because an additional 200-mg dose of carbamazepine was ineffective, we added a 400-mg daily dose of gabapentin. The pain attacks disappeared on the second day after initiating this treatment regime. On the fifth day, he complained of sudden onset of left lower abdominal pain, i.e., a pulling sensation that extended upward from the anus and lasted for $2 \mathrm{~h}$. A visceral crisis involving tabes dorsalis was suspected; therefore, we increased the gabapentin dosage to $600 \mathrm{mg}$ daily, which reduced his abdominal pain the next day. On the 14th day, the patient experienced complete relief from pain with an 800-mg dose of

K. Oshita $(\square) \cdot$ N. Saeki $\cdot$ H. Niinai · H. Hamada ·

M. Kawamoto

Department of Anesthesiology and Critical Care,

Hiroshima University Hospital, Hiroshima University,

1-2-3 Kasumi, Minami-ku, Hiroshima 734-8551, Japan

e-mail: oshitak@hiroshima-u.ac.jp gabapentin. Pain did not recur after carbamazepine was discontinued because of the toxic eruption on the 25th day. At the 1-year follow-up examination, he did not report recurring pain and complained only of slight dysesthesia in his right leg.

The pathophysiology of tabes dorsalis involves leptomeningeal inflammation and atrophy of the posterior columns of the spinal cord and posterior nerve roots [1]. In most patients, pain often persists after antibiotic treatment $[2,3]$ and should be treated as neuropathic rather than inflammatory pain. Very few studies have been performed on tabetic pain treatment, and there are only several case reports on pain modulation by carbamazepine or sodium valproate $[4,5]$. Our findings suggest that an alternative therapeutic regime involving gabapentin can effectively treat tabetic lightning pain.

Open Access This article is distributed under the terms of the Creative Commons Attribution Noncommercial License which permits any noncommercial use, distribution, and reproduction in any medium, provided the original author(s) and source are credited.

\section{References}

1. Dourmishev LA, Dourmishev AL. Syphilis: uncommon presentations in adults. Clin Dermatol. 2005;23:555-64.

2. Giménez-Roldán S, Martín M. Tabetic lightning pains: highdosage intravenous penicillin versus carbamazepine therapy. Eur Neurol. 1981;20:424-8.

3. Mao S, Liu Z. Neurosyphilis manifesting as lightning pain. Eur $\mathbf{J}$ Dermatol. 2009;19:504-6.

4. Ekbom K. Carbamazepine in the treatment of tabetic lightning pains. Arch Neurol. 1972;26:374-8.

5. Bowsher D, Lahuerta J. A case of tabes dorsalis with tonic pupils and lightning pains relieved by sodium valporate. J Neurol Neurosurg Psychiatry. 1987;50:239-41. 